\title{
Effects of Donor Age, Long-Term Passage Culture, and Cryopreservation on Tonsil- Derived Mesenchymal Stem Cells
}

\author{
Jin-Sik Choi ${ }^{a}$ Byung-Joo Lee ${ }^{a}$ Hee-Young Park ${ }^{a}$ Ji-Sun Song ${ }^{a}$ Sung-Chan Shin ${ }^{a}$ \\ Jin-Choon Lee ${ }^{b}$ Soo-Geun Wang ${ }^{a}$ Jin Sup Jung ${ }^{c}$ \\ aDepartment of Otorhinolaryngology-Head and Neck Surgery, Pusan National University School of \\ Medicine and Biomedical Research Institute, Pusan National University, Busan, Korea, 'Department \\ of Otorhinolaryngology-Head and Neck Surgery, Pusan National University School of Medicine and \\ Biomedical Research Institute, Pusan National University Yangsan Hospital, Yangsan, Kyeongnam, \\ Korea, 'Department of Physiology, School of Medicine, Pusan National University, Yangsan, Korea
}

\section{Key Words}

Tonsil-derived mesenchymal stem cells $\bullet$ Donor Age $\bullet$ Long-term passage $\bullet$ Cryopreservation

\begin{abstract}
Objectives: Human mesenchymal stem cells (MSCs) are efficacious in various cellular therapeutic applications and have been isolated from several tissues. Recent studies have reported that human tonsil tissue contains a new source of progenitor cells, potentially applicable for cell-based therapies. Information about the effects of donor age, long-term passage and cryopreservation are essential for clinical applications and cell-based therapies. Therefore, the authors investigated how the morphology, cell-surface markers, proliferation potential and differentiation capacity of tonsil-derived MSCs (T-MSCs) were affected by donor age, long-term passage, and cryopreservation. Materials and Methods: T-MSCs were isolated from tonsillar tissue of 20 patients undergoing tonsillectomy. Authors evaluated the effects of donor-age, long-term passage, and cryopreservation on the morphology, surface markers, proliferation potential and differentiation capacities of T-MSCs. Results: T-MSCs exhibited a fibroblast-like, spindle-shaped appearance. There were no significant morphological differences according to donor age, long-term passage or cryopreservation. T-MSCs isolated from donors of various ages were positive for markers CD90, CD44, and CD73, but negative for CD45, CD31, and HLA-DR. There were no significant differences in the expression of positive and negative surface markers as a function of donor age, longterm passage and cryopreservation. T-MSCs from different donor age groups showed similar proliferation potentials after passage 2 . After long-term passage and cryopreservation, there were no significant morphological differences. Cryopreservation did not affect the proliferation potential of T-MSCs, but there was a significant decrease in the proliferation potential in long-term passage T-MSCs (passage 15). The effect of donor age, long-term passage and cryopreservation on the in vitro adipogenic, osteogenic, and chondrogenic differentiation potential of T-MSCs was not significant. Conclusion: The effect of donor age, long-term

Byung-Joo Lee, MD, PhD

KARGER 125

Department of Otorhinolaryngology-Head and Neck Surgery,

Pusan National University School of Medicine and Medical Research Institute,

179 Gudeok-ro, Seo-gu, Busan, 602-739 (Republic of Korea)

Tel. +82-51-7335, Fax +82-51-246-8668, E-Mail voiceleebj@gmail.com
\end{abstract}




\section{Cellular Physiology Cell Physiol Biochem 2015;36:85-99

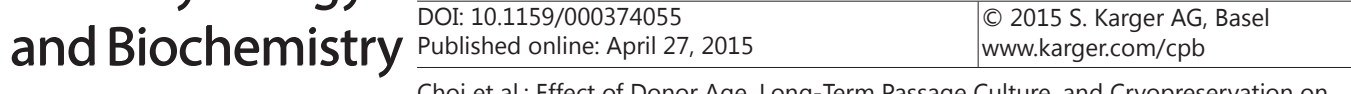 \\ Choi et al.: Effect of Donor Age, Long-Term Passage Culture, and Cryopreservation on T-MSCs}

passage culture, and cryopreservation on T-MSC properties are negligible, except for the proliferation capacity of long-term cultured T-MSCs. Therefore, T-MSCs are considered to be promising MSCs that can be used as future alternative sources for autologous or allogenic MSCs.

Copyright $(2015$ S. Karger AG, Basel

\section{Introduction}

Human mesenchymal stem cells (MSCs) have great therapeutic potential because of their ability to self-renew and differentiate into several cell types of the same mesenchymal lineages, including osteocyte, adipocyte and chondrocyte, as well as other lineages, such as neuronal and liver cells [1-4]. Although bone marrow is a good source of MSCs, bone marrow MSCs (BM-MSCs) have some limitations in the applications of cell-based therapies and regenerative medicine because the traditional procedure for procuring BM-MSCs is painful, frequently requiring general or spinal anesthesia, and may also yield low numbers of MSCs upon processing. Because of the low occurrence of MSCs in the bone marrow, in vitro expansion prior to clinical application is necessary. Therefore, a source of MSCs that is obtainable in large quantities under local anesthesia with minimal discomfort would be ideal. Several studies have reported the isolation of MSCs from various human adult and fetal tissues $[5,6]$.

The clinical application of MSCs requires large quantities of stem cells for injection or infusion. Because of the limitation of obtaining sufficient autologous MSCs within a limited time period, allogenic MSCs from multiple donor sites could constitute a valuable alternative source of stem cell-based therapy for clinical application. A prerequisite when considering allogenic MSCs for clinical applications is the properties of their immune modulatory activity under allogenic conditions.

Tonsillectomy and adenoidectomy for chronic tonsillar hypertrophy or chronic tonsillitis with adenoid vegetation are two of the most common operations in the ENT field. The human palatine tonsil and adenoid are readily accessible to otolaryngologists and are easily obtained by tonsillectomy or punch biopsy, particularly in young donors. Recent studies isolated tonsil-MSCs (T-MSCs) and adenoid-MSCs (A-MSCs) from their respective tissues and found that the morphology, cell-surface markers, proliferation potential and differentiation potential of T-MSCs and A-MSCs were similar to those of BM-MSCs $[5,7,8]$. Also, T-MSCs displayed the immunomodulatory effects that are associated with inhibiting T-cell proliferation, decreasing the production of soluble factors, and increasing HLA-G5 secretion in response to various stimuli [9]. These findings should contribute to increasing the feasibility of clinical applications by T-MSC injection or infusion in regenerative medicine and cell-based therapy applications.

Although T-MSCs can be obtained in adequate cell numbers from tonsillectomy in patients with chronic tonsillar hypertrophy and/or chronic tonsillitis and can be easily applied in cellbased therapy because they have immune modulatory activity similar to BM-MSCs, more information concerning the effect of donor age, long-term passage and cryopreservation on cell properties are essential for clinical applications and cell-based therapy. However, studies demonstrating the effect of donor age, long-term passage and cryopreservation on T-MSCs are limited. Therefore, the authors investigated how the morphology, cell surface markers, proliferation potential and differentiation capacity of T-MSCs were affected by donor-age, long-term passage, and cryopreservation.

\section{Materials and Methods}

Isolation and culture of T-MSCS

The protocol for MSC isolation from human tonsil tissue was approved by our institutional review board. Tonsils were obtained after informed consent from patients (5 to 54 years old) undergoing 


\section{Cellular Physiology Cell Physiol Biochem 2015;36:85-99 \\ \begin{tabular}{l|l} 
Dol: 10.1159/000374055 & $\begin{array}{l}\text { 2 2015 S. Karger AG, Basel } \\
\text { www.karger.com/cpb }\end{array}$ \\
\hline
\end{tabular} \\ Choi et al.: Effect of Donor Age, Long-Term Passage Culture, and Cryopreservation on T-MSCs}

tonsillectomy as a result of chronic tonsillar hypertrophy and/or chronic tonsillitis. Isolated T-MSCs were divided into four groups according to their donor age ranges: 0-10 years $(n=5), 11-20$ years $(n=5), 21-30$ years $(n=5)$, over 30 years $(n=5)$.

To isolate T-MSCs, tonsil tissues samples were washed with phosphate buffered saline (PBS) and digested at $37{ }^{\circ} \mathrm{C}$ for $30 \mathrm{~min}$ with $0.075 \%$ type I collagenase. The enzyme activity was neutralized with $\alpha$-modified Eagle's medium ( $\alpha$-MEM) containing 10\% fetal bovine serum (FBS). The samples were centrifuged at $1200 \times \mathrm{g}$ for $10 \mathrm{~min}$ and the pellet was incubated overnight at $37^{\circ} \mathrm{C}$ under $5 \% \mathrm{CO} 2 \mathrm{in}$ a control medium ( $\alpha$-MEM, 10\% FBS, $100 \mathrm{U} / \mathrm{ml}$ of penicillin and $100 \mu \mathrm{g} / \mathrm{ml}$ of streptomycin). Following incubation, the tissue culture plates were washed to remove any residual non-adherent cells and then maintained at 37 ${ }^{\circ} \mathrm{C}$ under 5\% CO2 in the control medium. One week later, when a monolayer of adherent cells had reached confluency, cells were trypsinized $(0.05 \%$ trypsin-EDTA; Sigma), re-suspended into media and subcultured at a concentration of 2,000 cells/cm2. The yield of non-adherent cells from each tonsil tissue ranged from 1 to $5 \times 109$. Their morphology was indicative of a hematopoietic origin. After multiple passages using multiple buffer washes and subsequent medium changes, approximately $0.1 \%-1 \%$ of the isolated cells were found to be adherent. T-MSCs were isolated as described previously [9].

Long-term passage culturing of T-MSCS

Adherent primary T-MSCs were expanded in culture and colonies started to form after 5-6 days of isolation. Medium refreshment was done twice weekly. When cells reached 80-90\% confluency, cells were detached with a $0.25 \%$ Trypsin/EDTA (Gibco) solution. Population doubling and cell viability were recorded. Then, cells were seeded into culture flasks at a density of $1.5 \times 103$ cells $/ \mathrm{cm} 2$ with DMEM-LG containing $10 \%$ MSC-FBS and incubated in a $37^{\circ} \mathrm{C}, 5 \% \mathrm{CO} 2$ incubator. Cells were subcultured every $4-5$ days and expanded up to 15 passages (P15).

Cryopreservation and thawing of T-MSCS

T-MSCs expanded over 4 weeks were cryopreserved in a $-200{ }^{\circ} \mathrm{C}$ liquid nitrogen (LN2) tank using StrataCooler Cryo preservation modules (Agilent Technologies, Santa Clara, CA, USA). At first, cells grown in culture dishes were washed with phosphate buffered saline (PBS, pH 7.4) and resuspended with cryomedium containing 50\% FBS, 40\% DMEM and 10\% dimethyl sulfoxide (DMSO). They were then aliquoted at a density of $2 \times 106$ cells/cryovial, placed at $-70^{\circ} \mathrm{C}$ for $24 \mathrm{~h}$, and transferred to and stored in an LN2 tank until use (9). After 14 days of cryopreservation, the cells were thawed in a $37^{\circ} \mathrm{C}$ water bath. The complete process of thawing, which should be rapid, usually took less than 2 min following a standard protocol. As soon as the cells were thawed, the cryovial was removed from the water bath and decontaminated by spraying with $70 \%$ ethanol. Thereafter, the cells were transferred to a centrifuge tube containing $8 \mathrm{~mL}$ of pre-warmed complete medium. After centrifugation, cells were resuspended with complete medium and dispensed into a 100-mm culture dish. In this study, the cryopreserved / thawed cells (CTC) were used and compared with fresh cultures.

\section{Colony numbers per adherent cell}

Nucleated cells from tonsils were plated at $1 \times 10^{5}$ and $1 \times 10^{6} / 60-\mathrm{cm}^{2}$ dishes and cultured for 7 days. The dishes were stained with $0.5 \%$ crystal violet and the colonies were counted. Colonies less than $2 \mathrm{~mm}$ in diameter and faintly stained colonies were ignored.

\section{Colony-forming assays}

At the second passage (P2), 100 cells were plated and cultured for 7 days in $60-\mathrm{cm}^{2}$ dishes. Cells were subsequently fixed with $4 \%$ paraformaldehyde, stained with $0.5 \%$ crystal violet in $4 \%$ paraformaldehyde for $5 \mathrm{~min}$, and washed twice with distilled water. The number of colonies was then counted.

\section{Cell proliferation assays}

Cell proliferation was evaluated by an MTT assay. T-MSCs were plated at a density of $1 \times 104$ cells/well in 24-well plates, and evaluated after 1, 2, 3 and 4 days of culture. After washing the cells, culture medium containing $0.5 \mathrm{mg} / \mathrm{ml}$ of MTT was added to each well. Cells were incubated for $2 \mathrm{~h}$ at $37^{\circ} \mathrm{C}$, the supernatant was removed and the formazan crystals that formed in viable cells were solubilized with $200 \mu \mathrm{l}$ of dimethyl sulfoxide. A $100 \mu \mathrm{l}$ aliquot of each sample was then transferred to 96-well plates and the absorbance of each 


\section{Cellular Physiology Cell Physiol Biochem 2015;36:85-99 \begin{tabular}{l|l} 
and Biochemistry Published onIIne: AprII Z7, 2015 & $\begin{array}{l}\text { C) 2015 S. Karger AG, Basel } \\
\text { www.karger.com/cpb }\end{array}$ \\
\hline
\end{tabular} \\ Choi et al.: Effect of Donor Age, Long-Term Passage Culture, and Cryopreservation on T-MSCs}

well was measured at $560 \mathrm{~nm}$ with an ELISA Reader equipped with XFLUOR4 software (version 4.51). This experiment was repeated four times. For the colony forming unit assay, T-MSCs were plated at a density of 100 cells in 60-mm plates and allowed to grow for 7 days. The cultures were terminated and stained with crystal violet for colony visualization. The colonies were counted manually under an inverted microscope.

\section{Flow cytometry analysis}

Flow cytometric analysis was used to characterize the phenotypes of the T-MSCs. At least 50,000 cells (in $100 \mu \mathrm{L}$ PBS/0.5\% BSA/2 mmol/L EDTA) were incubated with FITC-conjugated monoclonal antibodies against human CD45, CD31, HLA-DR, CD90, CD44 and CD73 (BD Biosciences). Labeled cells were analyzed by flow cytometry using a FACS Caliber flow cytometer equipped with Cell Quest Pro software (BD Biosciences).

\section{Multilineage differentiation}

Adipogenic differentiation. Adipogenic differentiation was induced by culturing T-MSCs for 3 weeks in adipogenic media ( $\alpha$-MEM supplemented with 10\% FBS, $1 \mathrm{uM}$ dexamethasone, $100 \mu \mathrm{g} / \mathrm{ml}$ 3-isobutyl-1 methylxanthine, $5 \mu \mathrm{g} / \mathrm{mL}$ insulin, and $60 \mu \mathrm{M}$ indomethacin) and assessed by oil red 0 (Sigma) staining as an indicator of intracellular lipid accumulation. Prior to staining, the cells were fixed for 15 min at room temperature in $70 \%$ ethanol. Cells were then incubated in $2 \%$ oil red 0 reagent for $1 \mathrm{~h}$ at room temperature. Excess stain was removed by washing with $70 \%$ ethanol and then distilled water to visualize lipid droplets.

Osteogenic differentiation. Osteogenic differentiation was induced by culturing T-MSCs for 3 weeks in osteogenic media ( $\alpha$-MEM supplemented with 10\% FBS, $0.1 \mathrm{mM}$ dexamethasone, $10 \mu \mathrm{M} \beta$-glycerophosphate, and $50 \mu \mathrm{g} / \mathrm{mL}$ ascorbic acid) followed by examining extracellular matrix calcification by alizarin red $\mathrm{S}$ (Sigma) staining. For alizarin red S staining, cells were fixed with $70 \%$ ethanol and washed with distilled water, then incubated in $2 \%$ alizarin red solution for $15 \mathrm{~min}$ at room temperature, followed by numerous washes with distilled water.

Chondrogenic differentiation. Chondrogenic differentiation was induced using the micromass culture technique. Briefly, $10 \mu \mathrm{L}$ of a concentrated MSC suspension $(3 \times 105$ cells $/ \mathrm{mL})$ was plated into the center of each well and allowed to attach at $37 \mathrm{C}$ for $2 \mathrm{~h}$. Chondrogenic media ( $\alpha$-MEM supplemented with 1\% FBS, $0.1 \mathrm{mM}$ dexamethasone (Sigma), $50 \mu \mathrm{g} / \mathrm{mL}$ ascorbic acid, insulin-transferrin-selenium (ITS+1; Sigma), and $10 \mathrm{ng} / \mathrm{mL}$ TGF- $\beta 1$ (Sigma)) was gently overlaid so as to not detach the cell nodules, and the culture was maintained in chondrogenic media for 4 weeks before analysis. Chondrogenesis was confirmed by immunohistochemistry for collagen type-II staining. For collagen type-II $\alpha 1$ staining, sections were blocked with $10 \%$ horse serum, incubated with purified anti-mouse collagen type-II $\alpha 1$ antibody (BD Bioscience) for $1 \mathrm{~h}$, and washed with PBS (pH 7.4). Antibody-bound cells were detected with a peroxidase substrate kit (Vectastain ABC kit; Vector Laboratories). Sections were washed, counterstained with hematoxylin, and examined by light microscopy.

\section{Real-time polymerase chain reaction}

Total cellular RNA was isolated from the cells and reverse transcribed using conventional protocols. The primer sequences used in the experiment were as follows: glyceraldehyde-3-phosphate dehydrogenase (GAPDH), 5' - GGA CTC ATG ACC ACA GTC CAT GCC - 3', 5' - TCA GGG ATG ACC TTG CC

CACA - 3'; lipoprotein lipase (LPL), 5' - GAG ATT TCT CTG TAT GGC ACC-3', 5' - CTG CAA ATG AGA CA

CTT TCT C - $3^{\prime}$; peroxisome proliferator-activated receptor-gamma (PPAR $\gamma$ ), $5^{\prime}$ - TGA ATG TGA AGC CC

ATT GAA -3', 5'-CTG CAG TAG CTG CAC GTG TT -3'; adipogenesis, alkaline phosphatase (ALP), 5' - TGG AGC TTC AGA AGC TCA ACA CCA -3', 5' - ATC TCG TTG TCT GAG TAC CAG TCC - $3^{\prime}$; osteocalcin (OC), $5^{\prime}$ - ATG AGA GCC CTC ACA CTC CTC - $3^{\prime}, 5^{\prime}$ - GCC GTA GAA GCG CCG ATA GGC - $3^{\prime}$; collagen type II $\alpha 1$ (COL2A1), 5' - GGA AAC TTT GCT GCC CAG ATG -3', 5' - TCA CCA GGT TCA CCA GGA TTG C - $3^{\prime}$; aggrecan (AGN), 5' - TGC GGG TCA ACA GTG CCT ATC $-3^{\prime}, 5^{\prime}$ - CAC GAT GCC TTT CAC CAC GAC $-3^{\prime}$. All of the primer sequences were determined using established GenBank sequences. Real-time quantitation was based on the LightCycler assay, using a fluorogenic SYBR Green I reaction mixture for PCR with a LightCycler instrument (Roche, Mannheim, Germany). The amplification program consisted of one cycle of $95^{\circ} \mathrm{C}$ with a 60 -s hold ("hot start"), followed by 40 cycles of $95{ }^{\circ} \mathrm{C}$ with a 0 -s hold, a specified annealing temperature with a 5 -s hold, $72{ }^{\circ} \mathrm{C}$ with 12 -s hold, and a specified acquisition temperature with a 2-s hold. All experiments were conducted three times, and negative and positive controls were included in all experiments. GAPDH mRNA was amplified as an 


\section{Cellular Physiology Cell Physiol Biochem 2015;36:85-99

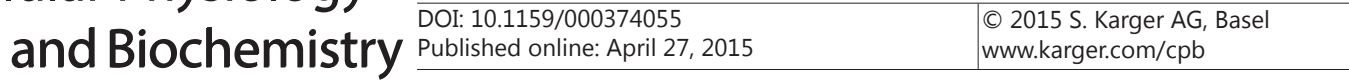 \\ Choi et al.: Effect of Donor Age, Long-Term Passage Culture, and Cryopreservation on T-MSCs}

Fig. 1. Representative flow cytometry graphs showing the cell surface epitopes. The T-MSCs were positive for CD90, CD44, and CD73, but negative for CD45, CD31, and HLA-DR. Three independent experiments were performed.

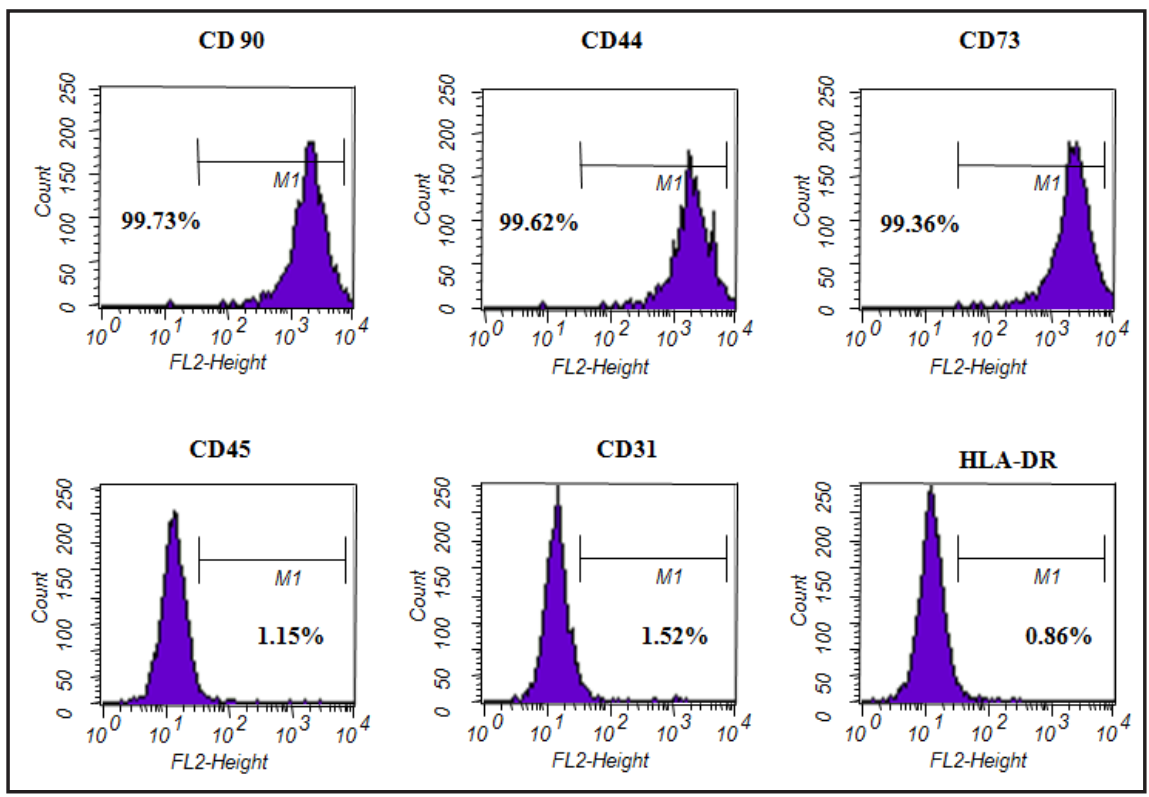

internal control. LightCycler software version 3.3 (Roche Diagnostics) was used to analyze PCR kinetics and calculate quantitative data. A standard curve generated in a separate run was loaded into runs of each sample (without standard curves). Each run included one sample of known concentration in the range covered by the standard curve, thus allowing the estimation of exact copy numbers by the second derivative maximum method. For each sample, copy numbers of target gene mRNA were divided by those of GAPDH mRNA to normalize for target gene mRNA expression, thus avoiding sample-to-sample differences in RNA quantity.

\section{Statistical analyses}

Effects of donor age, long-term passage and cryopreservation were analyzed by SPSS software (ver. 15.0; Chicago, IL, USA). Differences between levels of gene expression were evaluated using the KrusalWallis test. Probability values of $\mathrm{p}<0.05$ were considered statistically significant.

\section{Results}

\section{Effects of donor age on cell surface markers and proliferation ability}

Twenty donors were divided into four groups according to their age ranges: 0-10 years $(n=5), 11-20$ years $(n=5), 21-30$ years $(n=5)$, and over 30 years $(n=5)$. For characterization of the isolated T-MSCs from donors of various ages at P2, the expression of their cell surface antigens was examined by flow cytometry. T-MSCs from donors of various ages were positive for markers CD90, CD44, and CD73, but negative for CD45, CD31, and HLA-DR. A representative flow histogram is shown in Figure 1. There were no significant morphological differences according to donor age, long-term passage or cryopreservation. (Table 1).

To evaluate the proliferation potential, we measured the numbers of CFUs as a function of donor age group. There were significant increases according to donor age group when we measured the numbers of CFUs after culture of $1 \times 106$ cells of MSCs at passage 0 (Fig. 2A). The numbers of CFUs of T-MSCs increased according to a function of the inoculated cell number (Fig. 2B). However, T-MSCs isolated from different donor age groups showed similar proliferation potentials after passage 2 (Fig. 2C).

Effect of donor age on the in vitro differentiation potential of T-MSCs

We investigated the differentiation potential of isolated T-MSCs from donors of various ages. Morphologically, the formation of cytoplasmic lipid droplets was observed in T-MSCs after 1 week of adipogenic induction, as indicated by Oil Red 0 staining. There were no 
Table 1. Immunophenotypical characterization of isolated T-MSCs from donors of various ages

\begin{tabular}{lcccccc}
\hline Donor age (years) & \multicolumn{2}{c}{ Positive markers (\% gated) } & \multicolumn{3}{c}{ Negative markers (\% gated) } \\
& CD90 & CD44 & CD73 & CD45 & CD31 & HLA-DR \\
\hline$\leq 10$ & $99.8 \pm 0.13$ & $97.9 \pm 4.02$ & $99.6 \pm 0.35$ & $0.6 \pm 0.34$ & $0.7 \pm 0.44$ & $0.5 \pm 0.16$ \\
$11-20$ & $96.1 \pm 5.10$ & $99.4 \pm 0.49$ & $95.4 \pm 5.84$ & $1.2 \pm 0.94$ & $1.4 \pm 0.86$ & $0.9 \pm 0.42$ \\
$21-30$ & $99.7 \pm 0.18$ & $99.7 \pm 0.17$ & $99.6 \pm 0.16$ & $0.5 \pm 0.44$ & $0.2 \pm 0.21$ & $0.3 \pm 0.28$ \\
$>30$ & $99.2 \pm 0.87$ & $99.1 \pm 1.54$ & $99.5 \pm 0.29$ & $0.7 \pm 0.68$ & $0.6 \pm 0.52$ & $1.4 \pm 1.54$ \\
\hline
\end{tabular}

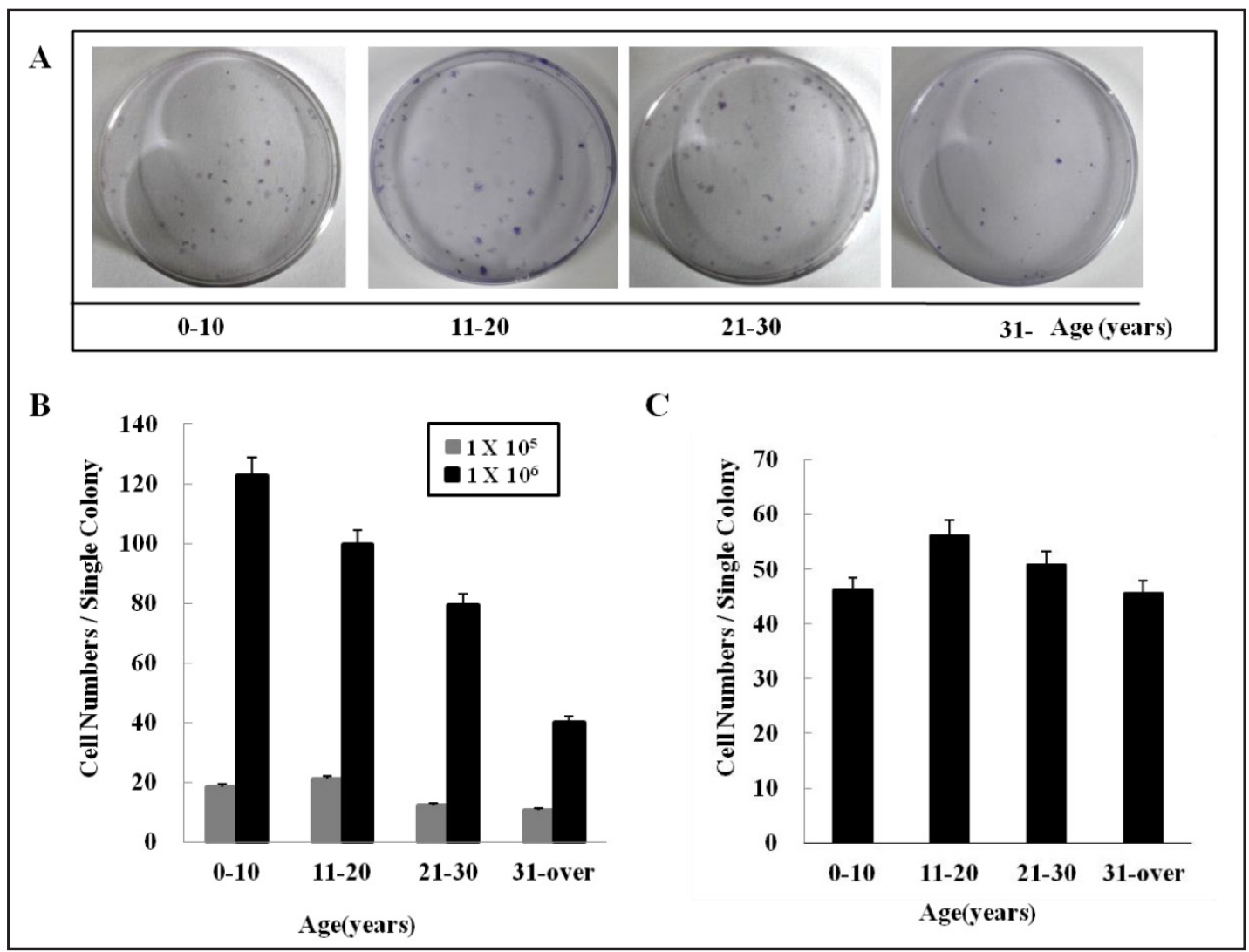

Fig. 2. The colony-forming unit and proliferation assays on T-MSCs. (A) Colony forming units (CFUs) at passage 0. (B) Significant increases were found as a function of donor age group when the numbers of CFUs after culturing of $1 \times 10^{6}$ MSCs at passage 0 were measured. (C) T-MSCs isolated from different donor age groups showed similar proliferation potentials after passage 2 . Data represent mean \pm SEM of 5 different experiments.

significant differences in Oil Red 0 staining of T-MSCs according to different donor age groups (Fig. 3A). Expression of LPL and PPAR $\gamma$ mRNA, which are markers of adipogenic differentiation, were enhanced after 21 days of T-MSC induction from different donor groups (Fig. 3B and C). The effect of donor age on the in vitro adipogenic differentiation potential of T-MSCs was not significant.

T-MSCs were observed to have a flattened morphology following osteogenic induction by osteogenic supplements and culture in expansion medium. Differentiation into osteoblasts was confirmed by positive Alizarin Red S staining. There were no significant differences in Alizarin Red S staining of T-MSCs according to different donor age groups (Fig. 4A). The mRNA expression of OC and ALP, which are markers of osteoblasts, increased in T-MSCs from different donor age groups (Fig. 4B and C). The effect of donor age on the in vitro osteogenic differentiation potential of T-MSCs was not significant.

\section{KARGER}



Cellular Physiology Cell Physiol Biochem 2015;36:85-99
\begin{tabular}{l|l} 
and Bioch 10.1159/000374055 & $\begin{array}{l}\text { O 2015 S. Karger AG, Basel } \\
\text { www.karger.com/cpb }\end{array}$ \\
\hline
\end{tabular}
Choi et al.: Effect of Donor Age, Long-Term Passage Culture, and Cryopreservation on T-MSCS

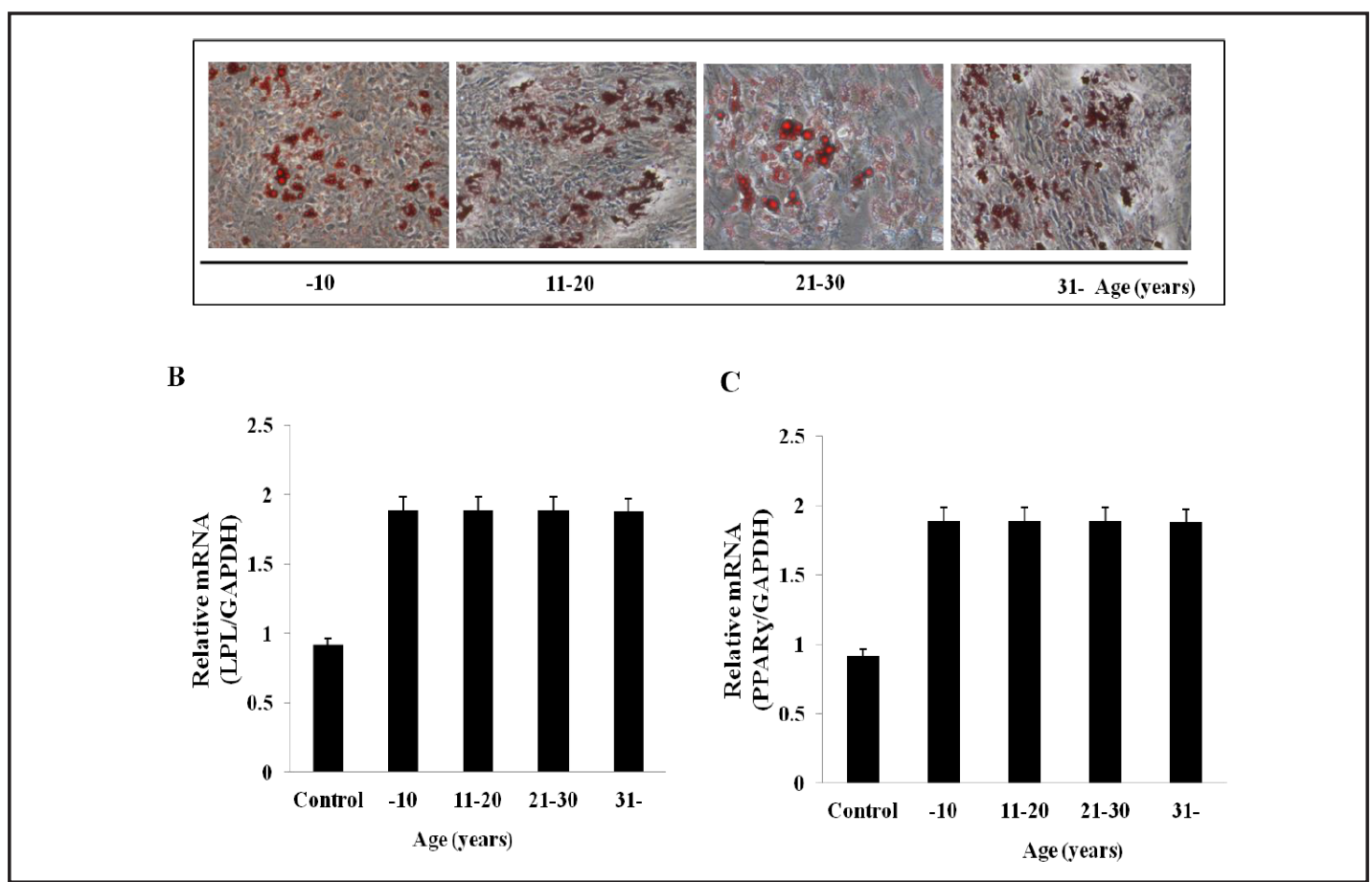

Fig. 3. Adipogenic differentiation potential of T-MSCs. (A) Lipid droplets were stained with Oil Red O. There were no significant differences in Oil Red O staining of T-MSCs according to different donor age groups. (B and C) Real-time reverse transcription polymerase chain reaction (RT-PCR) analysis of lipoprotein lipase (LPL) and proliferator-activated receptor-gamma (PPAR $\gamma$ ), showing enhanced expression after 21 days of induction of T-MSCs isolated from different donor age groups. The effect of donor age on Oil Red 0 staining and the expression of LPL and PPAR $\gamma$ in T-MSCs were not significant. The vertical axis of the graph shows the relative gene expression levels. Gene expression levels are presented as relative expressions over glyceraldehyde-3-phosphate dehydrogenase (GAPDH). Specific transcript levels of marker genes were normalized by comparison to those of GAPDH. Data represent mean \pm SEM of 5 different experiments.

Chondrogenic differentiation was assessed using high-density pellet cultures, which were maintained in chondrogenic medium. After 4 weeks of culture, the matrix-sulfated proteoglycan that accumulated during chondrogenic differentiation was determined by collagen type-II $\alpha 1$ staining. No apparent differences in shape or size were observed in the pellets of differentiated chondrocytes from the T-MSCs according to different donor age groups (Fig. 5A). The mRNA expression levels of COL2A1 and AGN, which are markers of chondrocytes, did not show any differences in the T-MSCs according to different donor age groups (Fig. 5B and C).

Effects of long-term passage and cryopreservation on the morphology of T-MSCS

We observed morphologic changes in T-MSCs according to long-term passage and cryopreservation. During early culturing (P2), T-MSCs exhibited a fibroblast-like, spindleshaped morphology. After long-term passage and cryopreservation, there were no significant morphological differences (Fig. 6).

Effects of long-term passage and cryopreservation on surface markers and proliferation potential of T-MSCS

T-MSCs were positive for markers CD90, CD44, and CD73, but negative for markers CD45, CD31, and HLA-DR. No significant differences were found in the expression of positive and negative surface markers according to long-term passage and cryopreservation (Table 2).

\section{KARGER}




\section{Cellular Physiology Cell Physiol Biochem 2015;36:85-99 \begin{tabular}{l|l} 
DOI: 10.1159/000374055 & (C) 2015 S. Karger AG, Basel
\end{tabular} \\ Choi et al.: Effect of Donor Age, Long-Term Passage Culture, and Cryopreservation on T-MSCs}

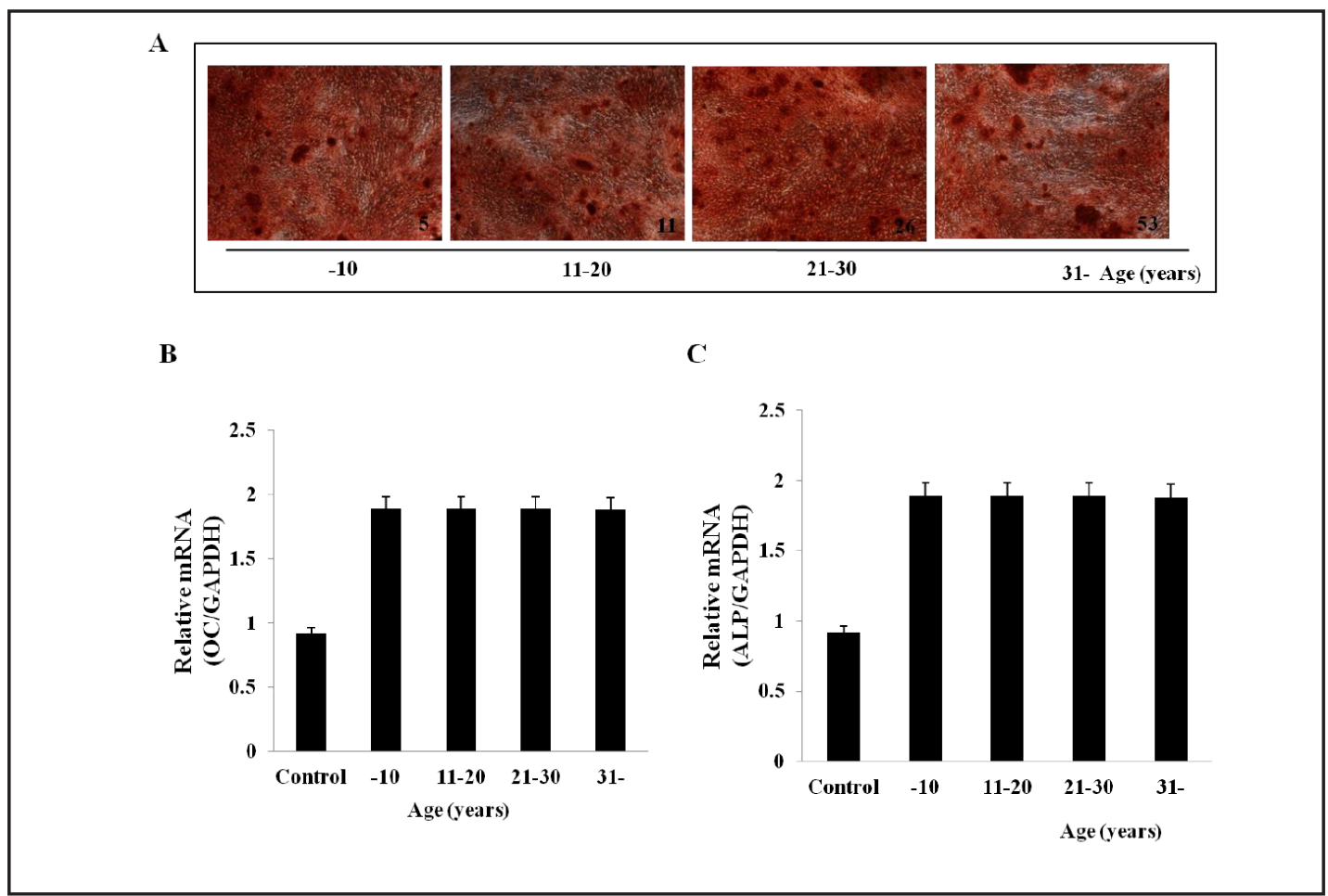

Fig. 4. Osteogenic differentiation potential of T-MSCs. (A) The mineralized matrix was stained with Alizarin Red S. There were no significant differences in Alizarin Red S staining of T-MSCs according to different donor age groups. (B and C) The mRNA expression of osteocalcin (OC) and alkaline phosphatase (ALP) are increased in T-MSCs from different donor age groups. The effect of donor age on Alizarin Red S staining and the expression of OC and ALP in T-MSCs were not significant. The vertical axis of the graph shows relative gene expression levels. Gene expression levels are presented as relative expressions over glyceraldehyde-3-phosphate dehydrogenase (GAPDH). Specific transcript levels of marker genes were normalized by comparison to those of GAPDH. Data represent mean \pm SEM of 5 different experiments.

To evaluate the proliferation potential according to long-term passage and cryopreservation, we measured the numbers of CFUs after seeding 100 cells. The numbers

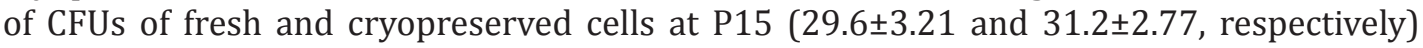
were significantly decreased compared to fresh and cryopreserved cells at P2 $(44.8 \pm 4.14$ and $45.0 \pm 2.12$, respectively) (Fig. 7A and B). Cryopreservation did not affect the number of CFUs of T-MSCs. However, long-term passage decreased the numbers of CFUs. In the MTT assay, cryopreservation did not affect the proliferation potential of T-MSCs, but there were significant decreases in the proliferation potentials of long-term passage T-MSCs (fresh and cryopreserved cells at P15) (Fig. 7C).

Effects of long-term passage and cryopreservation on the in vitro differentiation potential of T-MSCS

We investigated the differentiation potential of T-MSCs according to long-term passage and cryopreservation. Morphologically, the formation of cytoplasmic lipid droplets was observed in T-MSCs after adipogenic induction, as indicated by Oil Red 0 staining. There were no significant differences in Oil Red 0 staining of T-MSCs according to long-term passage and cryopreservation (Fig. 8A). The expression of LPL and PPAR $\gamma$ mRNA did not show significant differences after adipogenic induction of T-MSCs according to long-term passage and cryopreservation (Fig. 8B and C). The effects of long-term passage and cryopreservation on the in vitro adipogenic differentiation potential of T-MSCs were not significant.

Differentiation of T-MSCs into osteoblasts was confirmed by positive Alizarin Red S staining. There were no significant differences in Alizarin Red S staining of T-MSCs according KARGER 
A

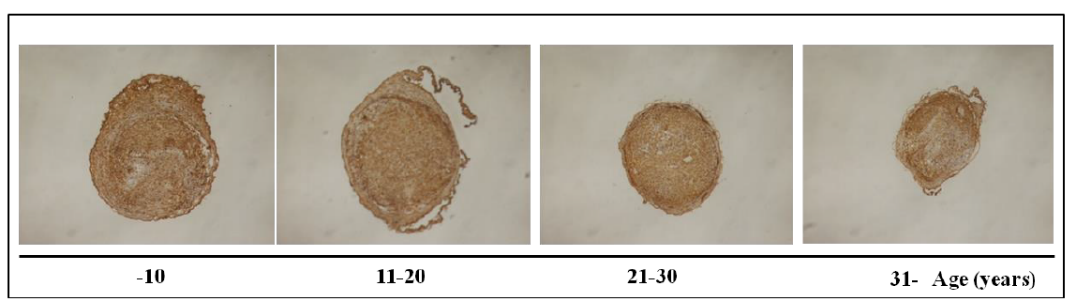

B

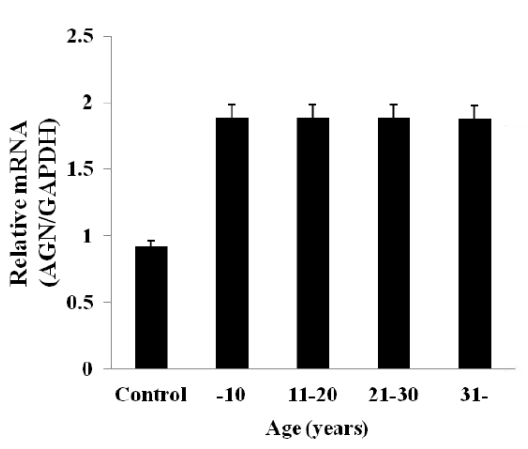

C

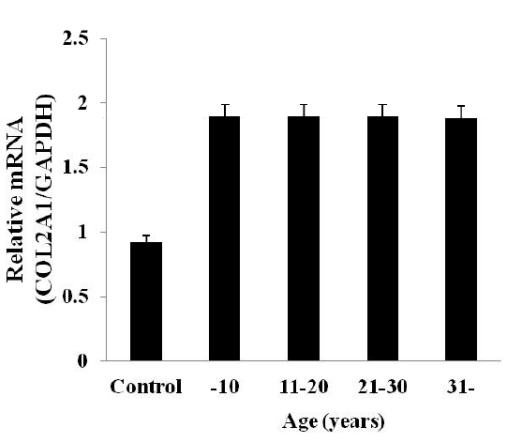

Fig. 5. Chondrogenic differentiation potential of T-MSCs. (A) Sulfated glycosaminoglycan-rich matrix was stained by immunohistochemistry for collagen type-II $\alpha 1$. There were no significant differences in collagen type-II $\alpha 1$ staining of T-MSCs according to different donor age group. (B and C) The mRNA expression of aggrecan (AGN) and collagen type-II $\alpha 1$ (COL2A1) was increased at 4 weeks in T-MSCs from different donor age groups. The effects of donor age on collagen type-II $\alpha 1$ staining and the expression of AGN and COL2A1 were not significant. The vertical axis of the graph shows relative gene expression levels. Gene expression levels are presented as relative expressions over glyceraldehyde-3-phosphate dehydrogenase (GAPDH). Specific transcript levels of marker genes were normalized by comparison to those of GAPDH. Data represent mean \pm SEM of 5 different experiments.

Fig. 6. Morphology of T-MSCs according to long-term passage and cryopreservation. T-MSCs had a fibroblast-like, spindle-shaped morphology. After long-term passage (P15) and cryopreservation (cryopreservation; P2 and P15), no significant morphological differences were observed.

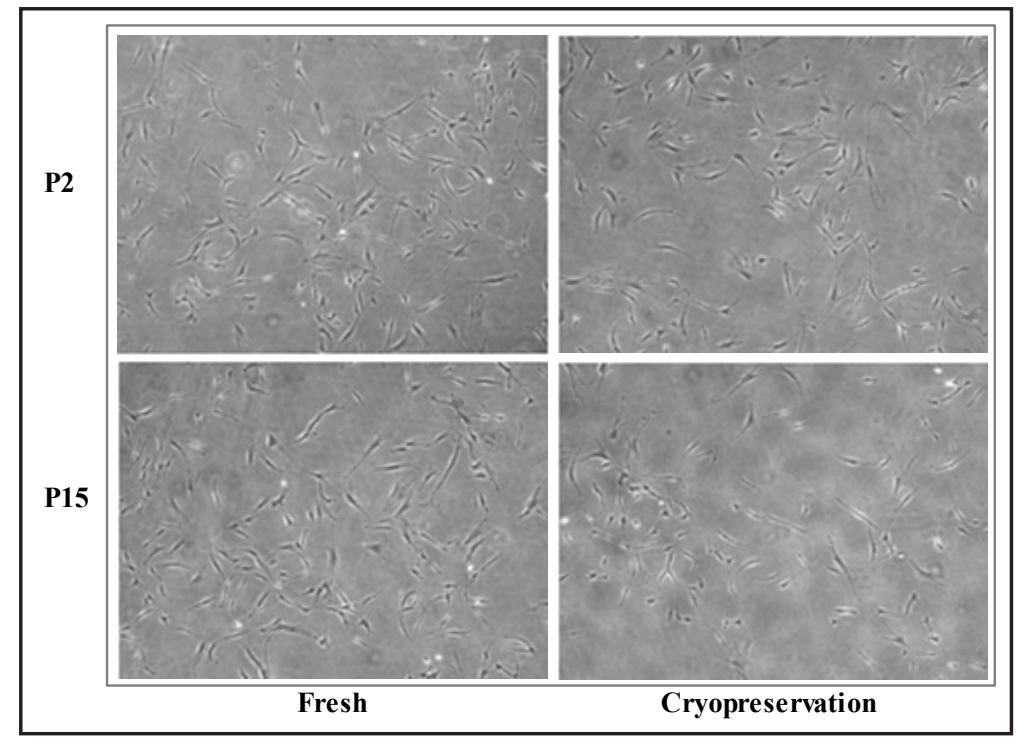

to long-term passage and cryopreservation (Fig. 9A). The mRNA expression of osteocalcin was decreased in T-MSCs after cryopreservation, but did not change after long-term passage (P15) (Fig. 9B). The mRNA expression of ALP did not show any significant differences in T-MSCs according to long-term passage and cryopreservation (Fig. 9C). The effects of longterm passage and cryopreservation on the in vitro osteogenic differentiation potential of T-MSCs were not significant.

\section{KARGER}


Table 2. Immunophenotypical characterization of isolated T-MSCs according to long-term culturing and cryopreservation

\begin{tabular}{lcccccc}
\hline & \multicolumn{3}{c}{ Positive markers (\% gated) } & \multicolumn{3}{c}{ Negative markers (\% gated) } \\
& CD90 & CD44 & CD73 & CD45 & CD31 & HLA-DR \\
\hline Fresh (P2) & $97.6 \pm 1.38$ & $98.4 \pm 1.73$ & $97.9 \pm 2.26$ & $1.5 \pm 0.76$ & $1.4 \pm 1.13$ & $0.8 \pm 0.83$ \\
Fresh (P15) & $97.7 \pm 2.35$ & $97.9 \pm 1.79$ & $98.2 \pm 1.42$ & $0.6 \pm 0.32$ & $0.4 \pm 0.23$ & $0.6 \pm 0.91$ \\
Cryopreservation (P2) & $98.5 \pm 0.99$ & $98.1 \pm 099$ & $98.8 \pm 0.46$ & $0.9 \pm 0.57$ & $0.5 \pm 0.32$ & $0.9 \pm 0.65$ \\
Cryopreservation (P15) & $98.5 \pm 0.68$ & $98.1 \pm 1.15$ & $98.5 \pm 0.73$ & $0.7 \pm 0.23$ & $0.5 \pm 0.35$ & $0.7 \pm 0.68$ \\
\hline
\end{tabular}

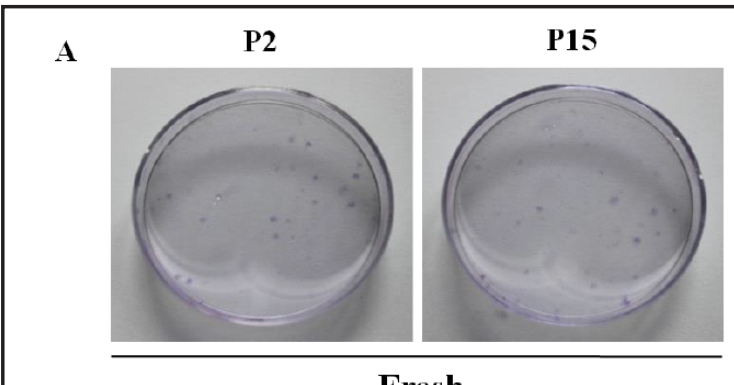

B

Fresh

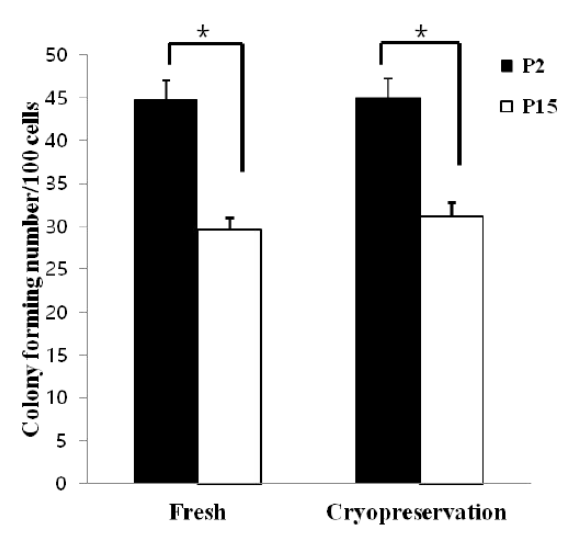

C

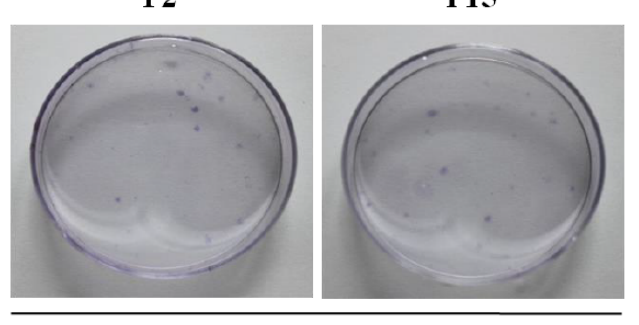

Cryopreservation

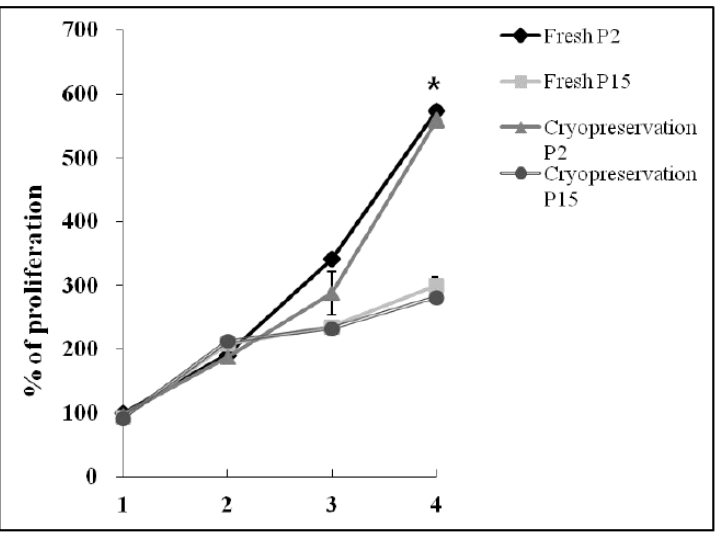

Fig. 7. Colony-forming unit and proliferation assays according to long-term passage and cryopreservation. The number of CFUs of fresh and cryopreserved cells at P15 are significantly decreased compared to fresh and cryopreserved cells at P2 (A and B). In the MTT assay (C), the proliferative ability at P15 is significantly decreased compared to P2, but cryopreservation did not affect the proliferation potential of the T-MSCs.

The chondrogenic differentiation potential was assessed, as described above. No apparent differences in shape or size were observed in the pellets of the chondrocytes differentiated from the T-MSCs as a function of long-term passage and cryopreservation (Fig. 10A). The mRNA expression of AGN and COL2A1 did not show any differences in T-MSCs as a function of long-term passage and cryopreservation (Fig. 10B and C).

\section{Discussion}

Although MSCs are an effective and promising tool for cell-based therapy and tissue engineering due to their ability to proliferate and differentiate into various cell types, MSC therapy cannot yet be used reliably because these cells show different behaviors across patients and/or MSC source. Although it is an advantage to use MSCs from young donor 


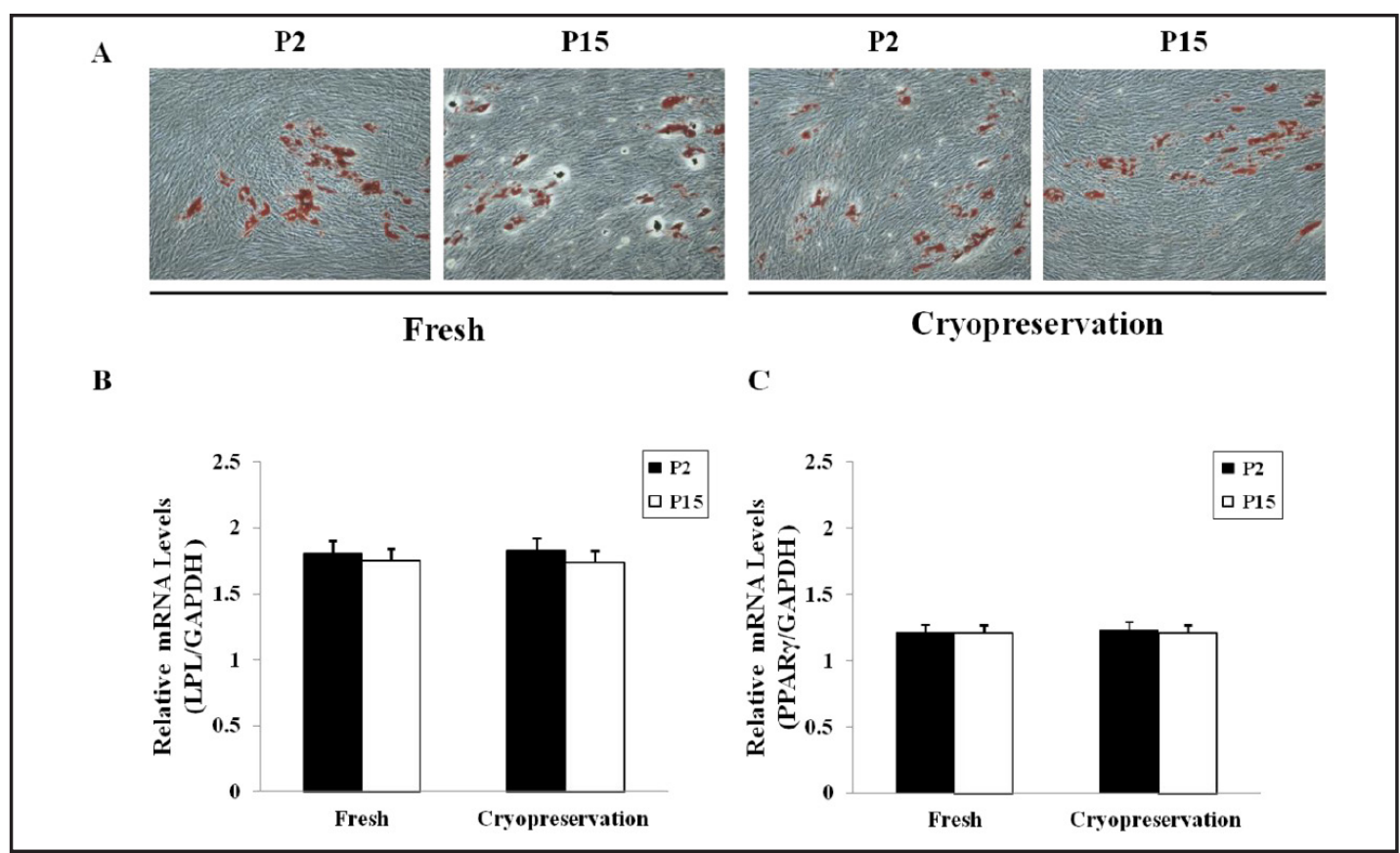

Fig. 8. Adipogenic differentiation potential of T-MSCs according to long-term passage and cryopreservation. (A) Lipid droplets were stained with Oil Red O. There were no significant differences in Oil Red 0 staining of T-MSCs according to long-term passage and cryopreservation. (B and C) The expression of LPL and PPAR $\gamma$ mRNA did not significantly differ after adipogenic induction of T-MSCs according to long-term passage and cryopreservation. The vertical axis of the graph shows relative gene expression levels. Gene expression levels are presented as relative expressions over glyceraldehyde-3-phosphate dehydrogenase (GAPDH). Specific transcript levels of marker genes were normalized by comparison to those of GAPDH. Data represent mean \pm SEM of 5 different experiments.

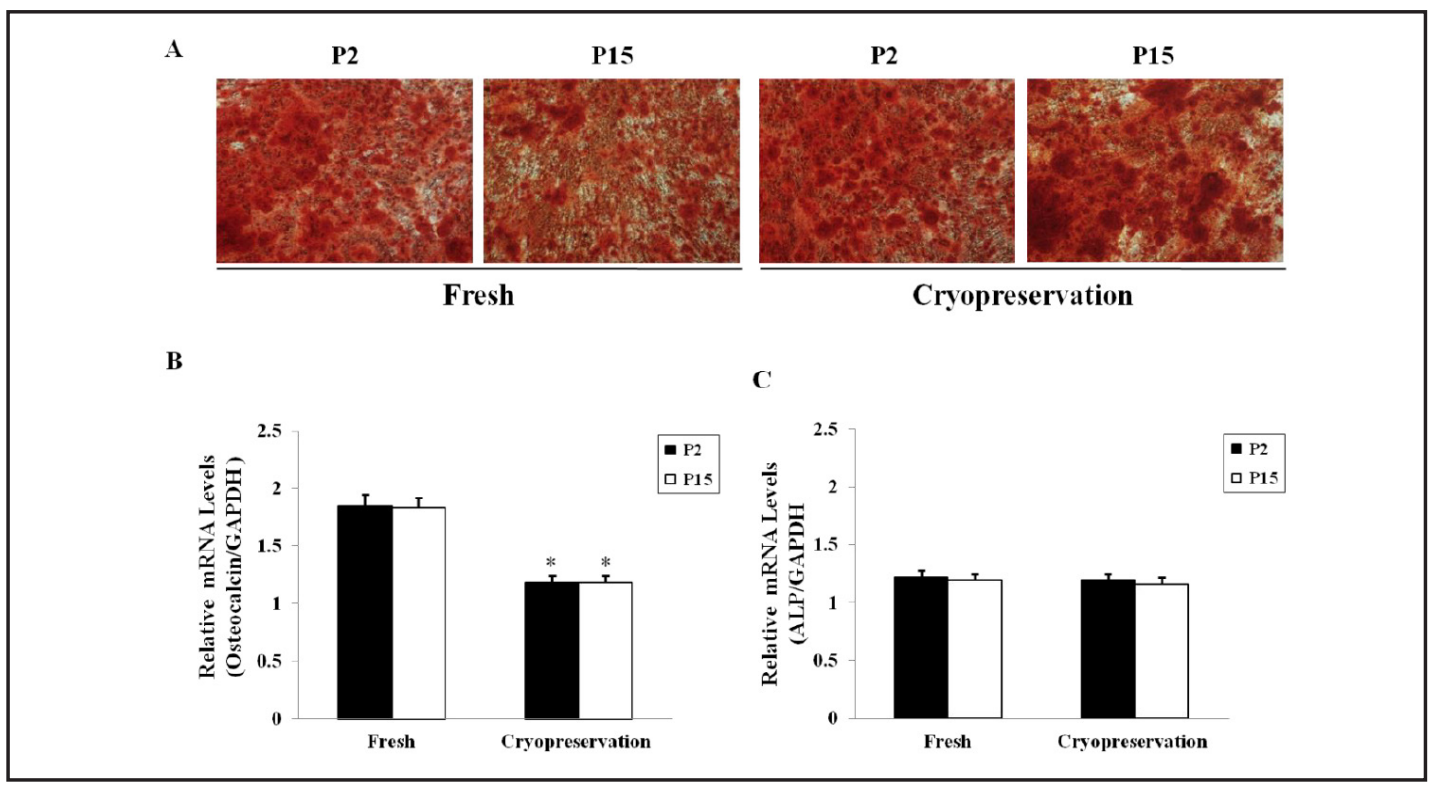

Fig. 9. Osteogenic differentiation potential of T-MSCs according to long-term passage and cryopreservation. (A) The mineralized matrix was stained with Alizarin Red S. There were no significant differences in Alizarin Red S staining of T-MSCs according to long-term passage and cryopreservation. (B) The mRNA expression of OC is decreased in T-MSCs after cryopreservation, but did not change after long-term passage (P15). (C) The mRNA expression of ALP did not show significant differences in T-MSCs according to long-term passage 


\section{Cellular Physiology Cell Physiol Biochem 2015;36:85-99 \begin{tabular}{ll|l} 
and Biochemistry Published onIIne: AprII 27, 2015 & $\begin{array}{l}\text { C) 2015 S. Karger AG, Basel } \\
\text { www.karger.com/cpb }\end{array}$ \\
\hline
\end{tabular} \\ Choi et al.: Effect of Donor Age, Long-Term Passage Culture, and Cryopreservation on T-MSCs}

and cryopreservation. The vertical axis of the graph shows relative gene expression levels. Gene expression levels are presented as relative expressions over glyceraldehyde-3-phosphate dehydrogenase (GAPDH). Specific transcript levels of marker genes were normalized by comparison to those of GAPDH. Data represent mean \pm SEM of 5 different experiments.

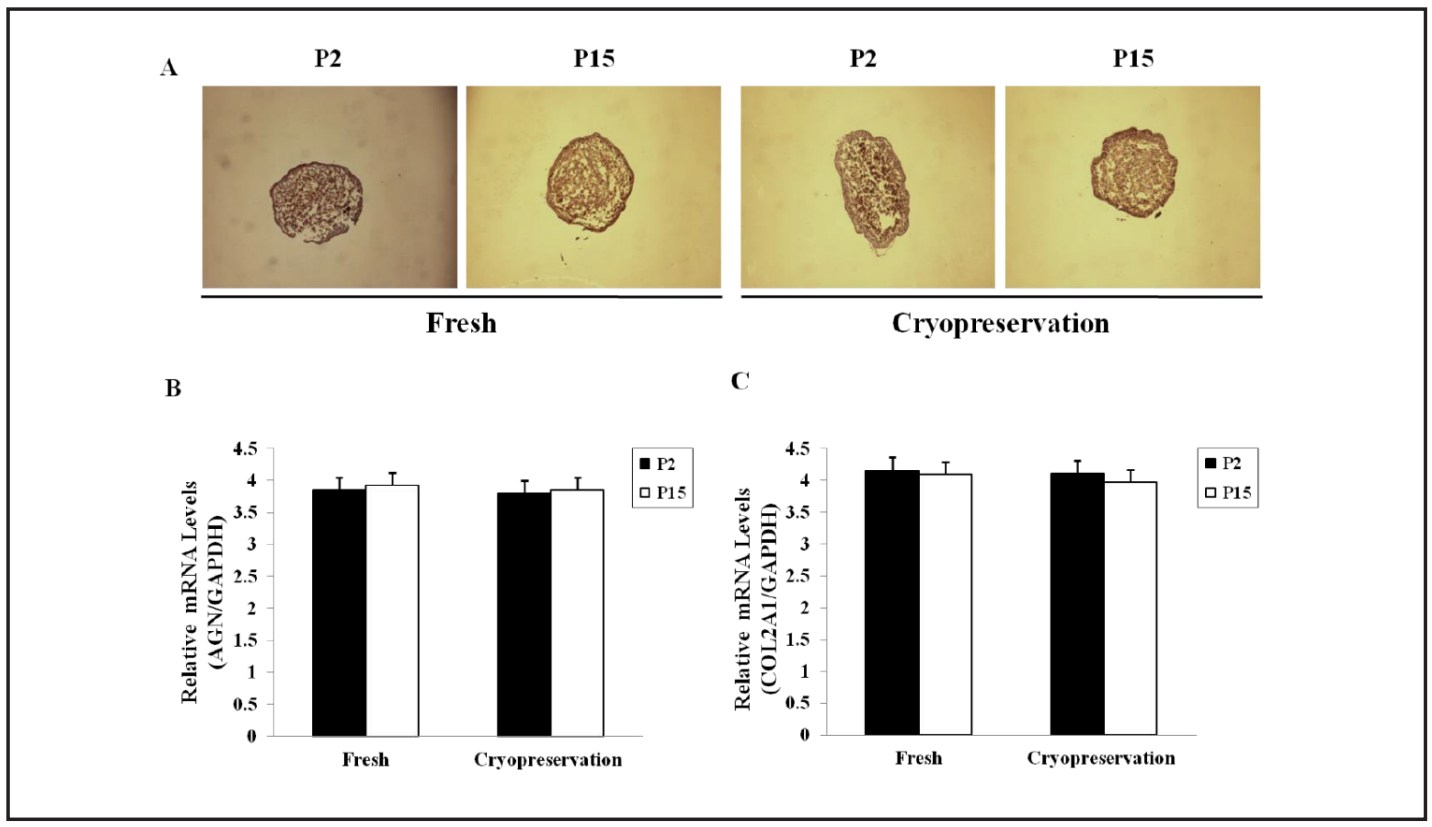

Fig. 10. Chondrogenic differentiation potential of T-MSCs according to long-term passage and cryopreservation. (A) Sulfated glycosaminoglycan-rich matrix was stained by immunohistochemistry for collagen type-II $\alpha 1$. There were no significant differences in collagen type-II $\alpha 1$ staining of T-MSCs according to longterm passage and cryopreservation. (B and C) The mRNA expression of AGN and COL2A1 did not significantly differ after adipogenic induction of T-MSCs according to long-term passage and cryopreservation. The vertical axis of the graph shows relative gene expression levels. Gene expression levels are presented as relative expressions over glyceraldehyde-3-phosphate dehydrogenase (GAPDH). Specific transcript levels of marker genes were normalized by comparison to those of GAPDH. Data represent mean \pm SEM of 5 different experiments.

age and of early passage culture in order to the clinical application of MSC therapy, many researchers have studied the effects of donor age, long-term passage, and cryopreservation on MSC properties, as well as proliferation and differentiation capacities in vitro. Although T-MSCs are reported as being a new source of MSCs for cell-based therapy or regenerative medicine, there are few studies demonstrating the effects of donor age, long-term passage, and cryopreservation on T-MSC properties.

The authors observed that T-MSCs isolated from different donor age groups had a similar spindle-shaped morphology during the initial and long-term phase. Although a significant decline in the numbers of CFUs was observed according to donor age group, there were no significant differences in cell numbers per single colony. These data indicated that age does not affect the proliferative ability of T-MSCs, although the density of T-MSCs declines with age. The donor age-related decline of CFU number is consistent with the results that were observed in BM-SCs by Stolzing et al. [10]. However, several researchers reported a reduction of the proliferative ability of MSCs according to different donor age $[11,12]$. The discrepancy among the studies may be related to the difference in the cell sources.

The effect of donor age on the differentiation ability of MSCs remains controversial. We found no significant differences in the specific cell surface marker profiles and T-MSC 


\section{Cellular Physiology Cell Physiol Biochem 2015;36:85-99

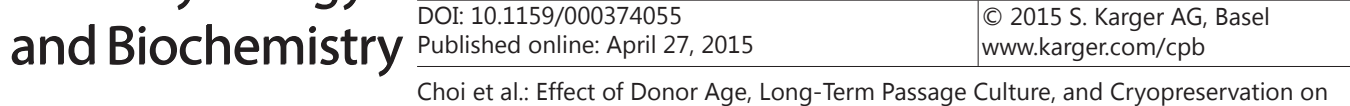 T-MSCs}

differentiation ability into osteogenic, adipogenic, and chondrogenic cells according to different donor age groups, which is consistent with previous findings in rats and humans that the differentiation potential of MSCs growing in vitro does not depend upon the age of the donor $[13,14]$. Stenderup et al. reported that MSCs from old donors showed no significant differences in differentiation ability into osteogenic and adipogenic tissues when compared with MSCs from young donors [15]. However, Baxter et al. found that MSCs from old donors showed significant decreases in osteogenic and adipogenic differentiation potential as compared to MSCs from young donors [16]. Zaim et al. reported an age-related decrease in osteoblastic but not chondrogenic differentiation potential in human BM-MSCs [12]. In previous studies about human donor age-related MSC properties, old donors were usually classified as being above 60 years of age. However, the donor age groups in this study were relatively younger than in previous studies. Because of the difference of donor age groups and cultivation conditions and intrinsic properties of MSCs, it is thought that there is no significant change in proliferation and differentiation ability in T-MSCs according to donor age. T-MSCs have the advantage that the donor age group is relatively younger than BMMSCs or adipose-derived MSCs, because tonsillectomies are usually performed in subjects aged 5-19 years.

In general, long-term passage culture-related changes in MSCs include increases in senescent cell number, as assessed morphologically, decreases in proliferation potential and loss of differentiation potential into osteogenic tissue $[17,18]$. When BM-MSCs were cultured to passage 12, morphological abnormalities, attenuated expression of specific surface markers and decreased proliferative ability appeared [19]. In our study, the proliferative ability of T-MSCs at P15 was significantly decreased compared to P2, which is in agreement with other previous studies. However, there were no significant differences in the morphology and expression of positive and negative surface markers according to long-term passage. These results are consistent with the results of Yu et al. who found no alterations in the expression of commonly measured surface antigens of T-MSCs from P3 to 15 [20]. The one advantage for the clinical application of T-MSCs is that MSC-specific surface marker characteristics are maintained up to P15.

Long-term passage cultures of MSCs shift the balance in favor of adipocyte differentiation at the expense of osteoblast differentiation, resulting in reduced bone formation and an increased risk for osteogenic disorders [21]. Zaim et al. have found a long-term passage culture-related decrease in osteoblastic but not chondrogenic differentiation potential in human BM-MSCs [12]. The change of chondrogenic potential during long-term passage cultures of MSCs has not been investigated in detail. In this study, despite long-term passage culturing until P15, there were no significant differences in the T-MSC differentiation potential into osteogenic, adipogenic and chondrogenic tissues at P15 as compared to P2. We did not found a shift in differentiation potential from an osteogenic to an adipogenic pattern during long-term passage culturing of T-MSCs in vitro. However, Yu et al. reported that the differentiation capacity of T-MSCs into either adipocytes or chondrocytes decreased dramatically in a passage-dependent manner, but the osteogenic differentiation potential of T-MSCs at P3 was similar to P15 [20]. Although the results of the osteogenic differentiation potential of T-MSCs at P15 is similar with our findings, the decrease in the adipogenic and chondrogenic differentiation potential at P15 are contradictory to our results. These conflicting results may be attributable to different experimental conditions.

MSCs from allogenic donors could constitute a valuable alternative source of stem cells for therapeutic applications because there are some limitations to obtaining sufficient autologous MSCs. T-MSCs displayed immunomodulatory effects that were associated with inhibiting T-cell proliferation, decreasing the production of soluble factors, such as tumor necrosis factor (TNF)- $\alpha$ and interferon (IFN)- $\gamma$, and increasing HLA-G5 secretion in response to various stimuli [9]. Therefore, if T-MSCs isolated from tonsillar tissue after tonsillectomy are cryopreserved in a liquid nitrogen tank, then T-MSCs can be used as autologous and/ or allogenic sources of MSCs in cell-based therapy and regenerative medicine in the future. When considering T-MSCs as allogenic and/or autologous sources of cell-based therapy after 


\section{Cellular Physiology Cell Physiol Biochem 2015;36:85-99

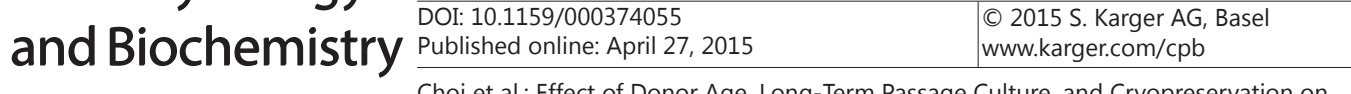 \\ Choi et al.: Effect of Donor Age, Long-Term Passage Culture, and Cryopreservation on T-MSCs}

cryopreservation, research concerning changes in T-MSC properties after cryopreservation and thawing is essential. In this study, we compared T-MSC properties before and after cryopreservation at P2 and P15 and found no significant differences in morphology, expression of cell surface markers, proliferation ability and differentiation potential into osteogenic, adipogenic and chondrogenic tissues. These results are consistent with those of Ryu et al. [8]. These results support the feasibility of the future use of T-MSCs as allogenic and/or autologous sources of cell-based therapy following cryopreservation.

In summary, no significant changes in T-MSC properties, including morphology, proliferation ability and differentiation ability according to donor age and cryopreservation were observed. Although the proliferation capacity of T-MSCs decreased significantly after long-term passage culture, there were no significant differences in morphology, cell surface markers, and differentiation potential according to long-term passage culture. An ideal MSC source for autologous and/or allogenic applications must fulfill the following criteria: ease of procuring cells with minimal patient discomfort, no donor age-dependence on MSC properties, and minimal processing time to obtain sufficient amounts of cells for clinical applications. In view of these criteria, T-MSCs are considered to be promising MSCs that can be used as future alternative sources for autologous or allogenic MSCs.

\section{Conclusion}

The effects of donor age, long-term passage culture, and cryopreservation on T-MSC properties are negligible, except for the proliferation capacity in long-term cultured T-MSCs. Therefore, T-MSCs are considered to be promising MSCs that can be used as future alternative sources for autologous or allogenic MSCs.

\section{Acknowledgements}

This work was supported by the Basic Science Research Program through a National Research Foundation of Korea (NRF) grant funded by the Ministry of Education, Science and Technology (No. NRF 2014R1A2A1A11052999) and (No. NRF-2012R1A1A4A01009912).

\section{Disclosure Statement}

The authors report no conflicts of interest. The authors alone are responsible for the content and writing of the paper.

\section{References}

1 Pittenger MF, Mackay AM, Beck SC, Jaiswal RK, Douglas R, Mosca JD, Moorman MA, Simonetti DW, Craig S, Marshak DR: Multilineage potential of adult human mesenchymal stem cells. Science 1999;284:143-147.

2 Dezawa M, Kanno H, Hoshino M, Cho H, Matsumoto N, Itokazu Y, Tajima N, Yamada H, Sawada H, Ishikawa H, Mimura T, Kitada M, Suzuki Y, Ide C: Specific induction of neuronal cells from bone marrow stromal cells and application for autologous transplantation. J Clin Invest 2004;113:1701-1710.

- 3 Dezawa M, Ishikawa H, Itokazu Y, Yoshihara T, Hoshino M, Takeda S, Ide C, Nabeshima Y: Bone marrow stromal cells generate muscle cells and repair muscle degeneration. Science 2005;309:314-317.

4 Pan RL, Chen Y, Xiang LX, Shao JZ, Dong XJ, Zhang GR: Fetal liver-conditioned medium induces hepatic specification from mouse bone marrow mesenchymal stromal cells: A novel strategy for hepatic transdifferentiation. Cytotherapy 2008;10:668-675. 


\section{Cellular Physiology Cell Physiol Biochem 2015;36:85-99

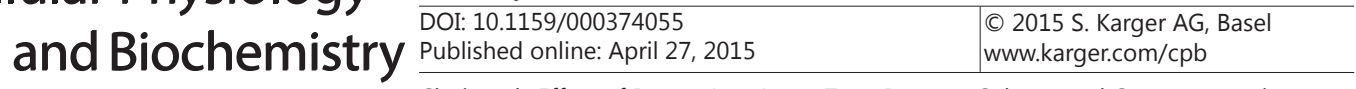 \\ Choi et al.: Effect of Donor Age, Long-Term Passage Culture, and Cryopreservation on T-MSCs}

5 Lee YS, Lee JE, Park HY, Lim YS, Lee JC, Wang SG, Lee BJ: Isolation of mesenchymal stromal cells (mscs) from human adenoid tissue. Cell Physiol Biochem 2013;31:513-524.

6 da Silva Meirelles L, Chagastelles PC, Nardi NB: Mesenchymal stem cells reside in virtually all post-natal organs and tissues. J Cell Science 2006;119:2204-2213.

7 Park MH, Yu Y, Moon HJ, Ko DY, Kim HS, Lee H, Ryu KH, Jeong B: 3d culture of tonsil-derived mesenchymal stem cells in poly(ethylene glycol)-poly(l-alanine-co-l-phenyl alanine) thermogel. Adv Healthc Mater 2014;3:1782-1791.

8 Ryu KH, Cho KA, Park HS, Kim JY, Woo SY, Jo I, Choi YH, Park YM, Jung SC, Chung SM, Choi BO, Kim HS: Tonsil-derived mesenchymal stromal cells: Evaluation of biologic, immunologic and genetic factors for successful banking. Cytotherapy 2012;14:1193-1202.

9 Lee YS PH, Lim YS, Lee JC, Wang SG, Jung JS, Lee BJ: Can the palatine tonsil be a source of mesenchymal stem cells with immunomodulatory property? Clinical Otolaryngol 2012;23:90-100.

10 Stolzing A, Jones E, McGonagle D, Scutt A: Age-related changes in human bone marrow-derived mesenchymal stem cells: Consequences for cell therapies. Mech Ageing Dev 2008;129:163-173.

11 Wagner W, Ho AD, Zenke M: Different facets of aging in human mesenchymal stem cells. Tissue Eng Part B Rev 2010;16:445-453.

12 Zaim M, Karaman S, Cetin G, Isik S: Donor age and long-term culture affect differentiation and proliferation of human bone marrow mesenchymal stem cells. Ann Hematol 2012;91:1175-1186.

13 Stenderup K, Rosada C, Justesen J, Al-Soubky T, Dagnaes-Hansen F, Kassem M: Aged human bone marrow stromal cells maintaining bone forming capacity in vivo evaluated using an improved method of visualization. Biogerontology 2004;5:107-118.

$>14$ Tokalov SV, Gruner S, Schindler S, Wolf G, Baumann M, Abolmaali N: Age-related changes in the frequency of mesenchymal stem cells in the bone marrow of rats. Stem Cells Dev 2007;16:439-446.

$\rightarrow 15$ Stenderup K, Justesen J, Clausen C, Kassem M: Aging is associated with decreased maximal life span and accelerated senescence of bone marrow stromal cells. Bone 2003;33:919-926.

16 Baxter MA, Wynn RF, Jowitt SN, Wraith JE, Fairbairn LJ, Bellantuono I: Study of telomere length reveals rapid aging of human marrow stromal cells following in vitro expansion. Stem Cells 2004;22:675-682.

17 Kretlow JD, Jin YQ, Liu W, Zhang WJ, Hong TH, Zhou G, Baggett LS, Mikos AG, Cao Y: Donor age and cell passage affects differentiation potential of murine bone marrow-derived stem cells. BMC Cell Biol 2008;9:60.

18 Fehrer C, Lepperdinger G: Mesenchymal stem cell aging. Exp Gerontol 2005;40:926-930.

19 Wagner W, Bork S, Horn P, Krunic D, Walenda T, Diehlmann A, Benes V, Blake J, Huber FX, Eckstein V, Boukamp P, Ho AD: Aging and replicative senescence have related effects on human stem and progenitor cells. PloS One 2009;4:e5846.

-20 Yu Y, Park YS, Kim HS, Kim HY, Jin YM, Jung SC, Ryu KH, Jo I: Characterization of long-term in vitro culture-related alterations of human tonsil-derived mesenchymal stem cells: Role for ccn1 in replicative senescence-associated increase in osteogenic differentiation. J Anat 2014;225:510-518.

-21 Kim M, Kim C, Choi YS, Kim M, Park C, Suh Y: Age-related alterations in mesenchymal stem cells related to shift in differentiation from osteogenic to adipogenic potential: Implication to age-associated bone diseases and defects. Mech Ageing Dev 2012;133:215-225. 